\title{
EL DIARIO DE APRENDIZAJES. UN INSTRUMENTO DE AYUDA EN LA AUTOEVALUACIÓN DEL ESTUDIANTE
}

\section{LEARNING'S DIARY. AN INSTRUMENT FOR THE STUDENT SELF-ASSESSMENT}

Rivera, Enrique \& Trigueros, Carmen

Facultad de Ciencias de la Educación, Universidad de Granada. España.

RIVERA, E. \& TRIGUEROS, C. El Diario de Aprendizajes. Un Instrumento de Ayuda en la Autoevaluación del Estudiante. Mot. Hum. 13(2); 110-117: 2012.

\section{RESUMEN}

Dentro del proyecto: "formar docentes, formar personas", el diario de aprendizajes es uno de los instrumentos creados para facilitar a nuestros estudiantes el proceso de reflexión crítica de cara a realizar su autoevaluación. Además es una herramienta fundamental para regular el proceso de enseñanza y aprendizaje. Facilita equilibrar la carga de trabajo del estudiante y valorar el impacto que las actividades propuestas para el desarrollo del proyecto están produciendo en el alumnado. En el artículo que se presenta, desde un análisis previo de la actual situación de la implantación del EEES y del ECTS, se realiza una aproximación al proyecto de intervención, y una descripción del instrumento. Para finalizar con una evaluación cualitativa del mismo desde la perspectiva de los docentes y de los estudiantes.

Palabras Clave: Evaluación formativa; Instrumentos de evaluación, Autoevaluación, Diario de aprendizaje, Tiempo no presencial del estudiante.

\begin{abstract}
Within the project "train teachers, to train people," the learning's diary is one of the tools developed to provide our students the process of critical thinking regarding to their self-assessment. It is also a fundamental tool to regulate the teaching and learning process. It makes easier to balance the load of workload and it assess the influence in the students of the activities proposed for the project. The present article, from a previous analysis of the current situation of the implementation of the EHEA and ECTS, is an approach to the intervention project, and a description of the instrument. Finishing with a qualitative assessment thereof from the perspective of teachers and students.
\end{abstract}

Keywords: Formative Assessment, Assessment tools, Self Assessment, Learning Journal, time student nonattendance. 


\section{INTRODUCCIÓN}

Llevamos cerca de una década inmersos en el nuevo lenguaje del Espacio Europeo de Educación Superior (EEES) y del European Credit Transfer System (ECTS), pero a día de hoy tendríamos que preguntarnos cuál ha sido el impacto real que ha tenido en la universidad española. ¿Qué ha quedado o está quedando de la declaración de intenciones que se realiza desde el Bologna Working Group on Qualifications Frameworks, 2005 (1).

Según su propia declaración, la educación superior debía de aspirar a formar profesionales para el mercado de trabajo, ciudadanos activos que participen de las decisiones de una sociedad democrática, lograr un buen desarrollo personal y lograr mantener una base amplia de conocimiento puntero. Como plantea Huber, al final del proceso las competencias se concentran en <<conocimiento y comprensión, aplicación de conocimiento, evaluación y decisión, comunicación y destrezas de aprendizaje -ninguna palabra más sobre el desarrollo personal, valores, sentimientos, responsabilidad y la sociedad democrática->> (2).

Podemos afirmar que los cambios han sido básicamente formales: organizacionales $y$ estructurales. Se ha dejado en un segundo plano la transformación de fondo; el cambio de la metodología de enseñanza. Tampoco pensemos que estamos ante grandes innovaciones, se trata de incorporar a la universidad $<<\operatorname{los}$ principios pedagógicos de los movimientos de renovación escolar que se generaron en Europa y en España desde la segunda mitad del siglo XIX > (3). Si analizamos brevemente estos principios pedagógicos y los traducimos al lenguaje ECTS, tenemos que centrarnos en facilitar la participación activa del estudiante en el proceso de enseñanza y aprendizaje propiciando que sea capaz de autorregular, construir, situar y socializar su aprendizaje, Shuell 1986 (4).

¿Dónde estamos fracasando? Según la opinión del profesorado en la excesiva masificación de las aulas. Teniendo en cuenta la limitación anterior, Se requiere un cambio de actitud y replantearse la necesidad de asumir el papel de facilitadores frente a la opción clásica de transmisores y fiscalizadores o calificadores (otros preferirían el concepto evaluadores), del conocimiento. Es preciso asumir que la enseñanza, además de vocacional es profesional y precisa de una formación inicial y permanente que facilite al docente estrategias pedagógicas para asumir el cambio.

El esfuerzo debe ser triple: del docente, pero también de la universidad que debe flexibilizar su organización académica y adaptar las infraestructuras a las demandas pedagógicas de las nuevas modalidades de enseñanza y de los estudiantes que deben abandonar la pasividad y tomar conciencia de que ellos son los protagonista de su aprendizaje. Sólo así podremos acercarnos a esa idea de formación integral a la que aludía el documento del (1), Rodríguez 2009 (5).

Las Facultades de Educación, debieran ser la vanguardia en cuanto a metodología docente se refiere. Sus propuestas de innovación tendrán que ir encaminadas en una doble dirección: preparar a los docentes responsables de la educación de las futuras generaciones del siglo XXI y ofrecer propuestas metodológicas transferibles a otros contextos de enseñanza universitaria.

La cristalización de la idea anterior debe materializarse, coincidiendo con la propuesta de Huber 2008 (2), en un triple objetivo: preparar a los estudiantes para que puedan asumir su responsabilidad en el ámbito laboral desde la adquisición de los conocimientos y competencias necesarias, construir ciudadanos que participen activamente en una sociedad democrática y ayudarles a que aprendan a asumir sus responsabilidades personales.

Tomando de referencia este triple objetivo, desde hace nueve años, venimos desarrollando en la Facultad de Educación donde trabajamos el proyecto de innovación "Formar docentes, Formar personas" (6), (7), (8) y (9). El proyecto parte de dos ideas claves: la educación no es neutral y cobra sentido si es proyectada en la comunidad. Desde la idea de facilitar este doble proceso, pensamos en la necesidad de construir una herramienta que, como complemento del diario personal, ayudara a nuestros estudiantes en la tarea de reflexión, crítica y toma de conciencia del proceso vivido. Es aquí donde nace el diario de aprendizajes, objeto de este trabajo. Nuestra 
RIVERA, E. \& TRIGUEROS, C. El Diario de Aprendizajes. Un Instrumento de Ayuda en la Autoevaluación del Estudiante. Mot. Hum. 13(2); 110-117: 2012.

preocupación no se focaliza en la línea marcada por De Lavigne 2003 (10) en la idea de equiparar los créditos con el trabajo de los estudiantes. Nos moveríamos en un híbrido entre dos de las tres propuestas que él realiza (impositiva $y$ compositiva). Pero tenemos que indicar que como objetivo secundario, también nos preocupa como docentes, poder ajustar al máximo la propuesta de las actividades de enseñanza y aprendizaje a los tiempos de trabajo de nuestros alumnos y pensamos que esta herramienta, podría ser un excelente instrumento para ayudarnos a realizar esta tarea de regulación.

Revisando los trabajos que se han venido realizando en estos últimos años sobre el tema, la mayoría de ellos plantean sus objetivos hacia la búsqueda del equilibrio perfecto entre tiempo de trabajo presencial y no presencial $(11,12$ y 13$)$. En otra dirección, bastante similar a la anterior, se mueven los estudios que buscan relacionar metodologías y tiempos de trabajo no presencial. La mayoría de ellos llega a una misma conclusión: que los tiempos de dedicación de los estudiantes suelen ser muy variables, dependiendo de múltiples factores, por lo que es complejo establecer una dedicación media a cada una de las tareas de aprendizaje propuestas. Esta afirmación queda patente en el estudio realizado por Julián, Zaragoza, Castejón y López 2010 (14).

Los estudiantes que participan se mueven en una horquilla de trabajo no presencial excesivamente amplia: entre 40 y 100 horas, teniendo además la sensación de que con una metodología activa y consecuente con los principios del ECTS, su dedicación aumenta considerablemente. En realidad no sobrepasan en la mayoría de los casos el máximo de horas que tendrían que dedicar. A esta misma conclusión llegan Barceló, Olivan, Asensio y González, 2007 (15), desde un estudio similar al anterior, dudando de la conveniencia de utilizar estos tiempos de dedicación como elemento de valoración del aprendizaje.

En la búsqueda de la relación perfecta entre dedicación presencial y no presencial del estudiante a la materia, Garmendia, Guisasola, Barragués, y Zuza 2006 (16) indican que la evaluación juega un papel importante en el ritmo de estudio y las horas de dedicación. Además, han podido comprobar que los estudiantes con mejores resultados académicos son los que mayor dedicación de tiempo no presencial a la materia declaran (algo obvio por otra parte). Por su parte, Palomares y Garrote 2009 (17), llegan a la conclusión que los tiempos no presenciales suelen superar a los presenciales, pero que adolecen en gran parte de un trabajo en colaboración, por lo que recomiendan potenciar este aspecto en el proceso. Por último, destacar el trabajo de LópezAguado 2010 (18) en el que construye un cuestionario para el estudiante desde el que poder valorar los tiempos de dedicación no presencial del estudiante desde seis factores: ampliación, colaboración, conceptualización, planificación, preparación de exámenes y participación. Al ser un trabajo de elaboración de cuestionario no se tienen datos de su aplicación.

Las investigaciones que hemos revisado anteriormente nos ayudan a dar respuesta a nuestro segundo objetivo marcado desde la utilización del diario de aprendizaje: regular los tiempos de dedicación no presencial del estudiante al proyecto, pero no nos ayudan en la idea primigenia: dotar a nuestros alumnos de una herramienta eficaz de cara a la toma de conciencia del trabajo realizado y la calidad del mismo.

\section{EL DIARIO DE APRENDIZAJES}

Es una herramienta digital, concretamente una hoja de cálculo de Excel, (Figura 1) que pretende ayudar al estudiante y al docente a tomar conciencia del proceso de enseñanza y aprendizaje vivido a lo largo del proyecto "Formar docentes, Formar personas". De cara al estudiante le va a permitir:

Controlar su presencia en las actividades presenciales programadas y realizar de forma simultánea una valoración cuantitativa de su participación.

Realizar el seguimiento de las actividades presenciales a las que asiste realizando una breve valoración cualitativa y cuantitativa de la misma y del tiempo invertido en su realización.

Tomar conciencia del tiempo invertido en la realización de las actividades no presenciales que demanda el proyecto y realizar una evaluación cualitativa y cuantitativa de las mismas. 
RIVERA, E. \& TRIGUEROS, C. El Diario de Aprendizajes. Un Instrumento de Ayuda en la Autoevaluación del Estudiante. Mot. Hum. 13(2); 110-117: 2012.

Valorar de forma cuantitativa y cualitativa las tutorías realizadas y realizar un seguimiento del tiempo dedicado a ellas.

Lo que está facilitando al estudiante esta herramienta es poder tomar conciencia del trabajo realizado, facilitando la elaboración del informe final de autoevaluación que realiza al final del proyecto, en el que debe identificar sus progresos en el desarrollo de las competencias instrumentales y personales que se plantean desde el proyecto.

En cuanto al docente, el diario de aprendizaje le va a permitir conocer la opinión y valoración de los estudiantes respecto a las actividades de enseñanza $\mathrm{y}$ aprendizaje planteadas en el proyecto. Esto significa que podemos valorar la carga de trabajo no presencial y el equilibrio con las tareas que complementan la formación en los tiempos de presencialidad. Además facilita analizar de forma independiente cada una de las propuestas, lo que permite poder modificar aquellas que no se han ajustado a los intereses o demandas de los estudiantes, especialmente en el capítulo de las lecturas básicas.

Elementos del diario Como podemos observar en la figura 1, el diario de aprendizajes se organiza en tres grandes apartados, precedidos de un espacio previo de identificación del estudiante y medias globales de los apartados del mismo.

Apartado 1. Controlo mi asistencia a clase. Calendario de los días lectivos en los que se desarrolla el proyecto, en el que el estudiante debe indicar con una valoración de 0 a 10 puntos su participación en las actividades de enseñanza y aprendizaje desarrolladas. La idea de la participación que deseamos asuman nuestros alumnos es la que expresamos en la "Guía para elaborar el diario de aprendizajes" (GEDA) cuando hacemos mención a que $<<\ldots$ para nosotros no basta con "estar", atender, hacer las prácticas, además hay que aportar opiniones, críticas, propuestas, etc.>>.

\section{Apartado 2. Valoración de las actividades presenciales.}

Este apartado, a su vez, se subdivide en dos subapartados; el primero dedicado a las actividades de enseñanza y aprendizaje realizadas en grupo de teoría o práctica y el segundo pensado para que los estudiantes valoren y controlen su presencia en las tutorías, grupales o individuales que realicen a lo largo del proyecto.

Apartado 3. Controlo la realización de otras
actividades realizadas en grupo
$\begin{array}{llll}\text { individualmente fuera del aula } & \text { Lo que le }\end{array}$ pedimos al estudiante queda claro en la GEDA cuando le indicamos que debe $<<\ldots$ anotar todo, por lo que te aconsejamos que pongas el "crono" en marcha cuando te sientes a leer, a realizar un guión de lectura, preparar una propuesta, te reúnas con tu grupo, pones al día tus diarios etc $>\mathrm{La}$ idea es que para cada una de las actividades de aprendizaje propuestas abra una fila en el diario, de esta forma le permite y nos permite, tener una idea clara del tiempo dedicado y lo que ha significado para su proceso de aprendizaje. Aquí es importante que se diferencien con claridad las actividades realizadas a nivel individual o en colaboración con su grupo de trabajo.

Si nos fijamos en la figura 1, podemos observar que tanto en los apartados dos y tres el estudiante tiene que completar cinco epígrafes. El primero de ello, la fecha, queda definida previamente por el docente en el epígrafe $2 \mathrm{~A}$, ya que, salvo imprevistos, son coincidentes con los días de clase de las asignaturas. Esto cambia en los apartados 2B y 3, ya que no pueden ser previstos de forma anticipada. En el segundo epígrafe le pedimos al estudiante que nos contabilice el tiempo dedicado a la realización de la actividad, se lo pedimos en minutos para facilitar el cálculo posterior de los promedios.

El tercer epígrafe se dedica a "titular" la actividad con un máximo de 15 palabras.

El cuarto está pensado para que el estudiante realice una valoración cualitativa de la actividad realizada, en un máximo de dos líneas le pedimos que exprese su opinión, para finalmente, en el epígrafe quinto, debe traducir la evaluación cualitativa anterior en un valor numérico de 0 a 10. Dentro de los cambios a introducir para el próximo curso, estamos valorando sustituir en el cuarto la descripción valorativa, por la expresión de cinco adjetivos que definan lo que opina sobre la actividad realizada. 
RIVERA, E. \& TRIGUEROS, C. El Diario de Aprendizajes. Un Instrumento de Ayuda en la Autoevaluación del Estudiante. Mot. Hum. 13(2); 110-117: 2012.

ENSEÑANZA DE LA EDUCACIÓN FISICA EN LA EDUCACIÓN PRIMARIA DIARIO DE APRENDIZAUES

\begin{tabular}{|c|c|c|c|c|c|c|}
\hline \multirow[b]{2}{*}{ REALIZADO POR: } & & & \\
\hline & & & Grupo: & & & \\
\hline & \multicolumn{2}{|c|}{ PROMEDIOS } & \multicolumn{4}{|c|}{ Tiempos de Dedicación } \\
\hline Apartado 1 & Apartado 2 & Apartado 3 & Presenc. & 0,0 & No Presenc. & 0,0 \\
\hline & 2A: Actv. & & & & & \\
\hline & 28: Tutorias & & & & & \\
\hline
\end{tabular}

Apartado 1: CONTROLO MI ASISTENCIA Valora de 0 a 10 puntos tu participación en las clase del día (si no asistes a clase ese día debes poner un 0 A CLASE. en la casilla correspondiente)

\begin{tabular}{|l|l|l|l|l|l|l|l|l|l|l|l|l|l|l|l|l|l|l|l|l|l|l|l|l|l|l|l|l|l|l|l|}
\hline Día & 1 & 2 & 3 & 4 & 5 & 6 & 7 & 8 & 9 & 10 & 11 & 12 & 13 & 14 & 15 & 16 & 17 & 18 & 19 & 20 & 21 & 22 & 23 & 24 & 25 & 26 & 27 & 28 & 29 & 30 & 31 \\
\hline Febrero & & & & & & & & & & & & & & & & & & & & & & & & & & & & & & & \\
\hline Marzo & & & & & & & & & & & & & & & & & & & & & & & & & & & & & & & \\
\hline Abril & & & & & & & & & & & & & & & & & & & & & & & & & & & & & & & \\
\hline Mayo & & & & & & & & & & & & & & & & & & & & & & & & & & & & & & & \\
\hline Junio & & & & & & & & & & & & & & & & & & & & & & & & & & & & & & & \\
\hline
\end{tabular}

Apartado 2A: VALORACIÓN DE LAS ACTIVIDADES PRESENCIALES REALIZADAS (hace referencia a todas las actividades que has realizado y en las que la profesora ha estado presente. Clases teórica, clase práctica, talleres, conferencias, ...). Rellena correctamente todos los apartados, teniendo en cuenta que: El tiempo invertido se debe poner en minutos. La "breve descripción de la actividad" no debe ocupar: más de 15 palabras. Lo "más significativo de la actividad" no debe ocupar más de 40 palabras. En la "valoración de la actividad" se valora la actividad, no tu participación o actuación en ella. NO RELLENAR LOS APARTADOS DE LOS DÍAS EN LOS QUE NO SE HA ASISTIDO A LA CLASE.

\begin{tabular}{|l|l|l|l|l|}
\hline Fecha & $\begin{array}{c}\text { Tiempo } \\
\text { Invertido }\end{array}$ & Breve descripción de la actividad & Cita lo más significativo para ti de la actividad & $\begin{array}{c}\text { :tar. } \\
\bullet .10\end{array}$ \\
\hline & & & & \\
\hline & & & & \\
\hline
\end{tabular}

Apartado 2B: VALORACIÓN DE LAS TUTORÍAS REALIZADAS. Indicar en este apartado las tutorias realizadas y su valoración, tanto a nivel grupal como a nivel infividual.

\begin{tabular}{|c|c|c|c|c|}
\hline Fecha & $\begin{array}{c}\text { Tiempo } \\
\text { Invertido }\end{array}$ & Breve descripción de la actividad & Cita lo más significativo para ti de la actividad & $\begin{array}{l}\text { Peler. } \\
\text { Porres. } \\
\text { ot }\end{array}$ \\
\hline Tutoria 1 & & & & \\
\hline Tutoría 2 & & & & \\
\hline
\end{tabular}

Apartado 3: CONTROLO LA REALIZACIÓN DE OTRAS ACTIVIDADES REALIZADAS EN GRUPO O INDIVIDUALMENTE FUERA DEL AULA. (REalización de trabajos, proyecto, lecturas, intervención en Centros, reuniones de grupo ...). Igual que en el apartado anterior no sobrepasar las 10 palabras y las 40 palabras en los apartados de descripción y significatividad de la actividad.

\begin{tabular}{|c|c|c|c|c|}
\hline Fecha & $\begin{array}{c}\text { Tiempo } \\
\text { Invertido }\end{array}$ & Breve descripción de la actividad & Cita lo más significativo para ti de la actividad & 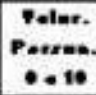 \\
\hline & & & & \\
\hline & & & & \\
\hline
\end{tabular}

Figura 1: Ejemplo de Diario de aprendizajes

\section{EVALUANDO LA HERRAMIENTA}

Para nosotros, desde nuestra perspectiva de docentes, es un instrumento de mucha utilidad para realizar el seguimiento de los alumnos a lo largo del desarrollo del proyecto, de hecho, es uno de nuestros principales referentes para poder visualizar de forma rápida su proceso de aprendizaje. Como instrumento de evaluación del proyecto nos aporta una información muy valiosa sobre las actividades de enseñanza y aprendizaje propuestas, ya que, al final de la misma podemos ver si nuestras expectativas han sido corroboradas desde la opinión del alumnado. 
RIVERA, E. \& TRIGUEROS, C. El Diario de Aprendizajes. Un Instrumento de Ayuda en la Autoevaluación del Estudiante. Mot. Hum. 13(2); 110-117: 2012.

En cuanto a las percepciones que expresan los estudiantes sobre el diario de aprendizaje, a través del documento de evaluación final de la propuesta, podemos ver cómo en primer lugar se produce una sensación de saturación, especialmente al principio, cuando tienen que enfrentarse a un proyecto que les demanda una dedicación diaria y la necesidad de tener que preparar una o dos actividades de aprendizaje cada semana. Incluso, desde la pérdida del objetivo global del proyecto desde la visión sesgada de lo particular, les lleva a pensar que su utilidad es dudosa, pero reconocen que $<<\ldots$ al final del proyecto ha sido lo que de una forma rápida y clara me ha permitido ver lo que he aprendido y que he hecho en cada momento, por eso me parece un complemento del diario personal.>> (MS76).

La falta de perspectiva que tienen los estudiantes cuando comienzan el proyecto, es lo que les lleva a ver de inicio el diario de aprendizaje como una carga más que como una ayuda, pero una vez completado el camino y reflexionando sobre lo vivido a lo largo del mismo les lleva a reconocer que:

$<<\ldots$ el gran acierto de estas asignaturas ha sido que se obligue en los alumnos a que mantenga cierta constancia en el trabajo, es decir que se realicen lecturas básicas en unos tiempos límites para poder tratar ciertos temas en unos días específicos, la realización de los retos tanto individuales como grupales y por supuesto el diario de aprendizaje y el blog (optativo) que nos obliga a desarrollar día a día un seguimiento para apuntar lo más significativo de cada día.>> (HS76) Efectivamente, $<<\ldots$ cuesta trabajo llevarlo al día [pero lo identifican como positivo] ... porque así hay un cierto control del trabajo realizado por cada alumno.>> (MN50). De la cita de este estudiante lo que nos preocupa es, que a pesar de ser un proyecto abierto, donde la responsabilidad pasa a manos del alumnado, incluidos los de la evaluación y calificación, mantienen la idea de que el control debe estar en manos de la autoridad (el docente), sin haber llegado a la convicción de que ellos son su propia autoridad. Lo que nos están diciendo entre líneas un sector de nuestros estudiantes, no es otra cosa que no han sabido romper la barrera de la motivación extrínseca y ser capaces de llegar a un estado de motivación intrínseco, $(19,20,21)$, que les haga ver en la oportunidad de formarse como docentes y personas que les ofrece el proyecto, al margen de la calificación.

$<<\ldots$ yo sabía desde el primer momento que al final de cuatrimestre, en función de mi trabajo, yo me llevaría la nota que me mereciera, la nota justa, sin tener que depender del "cara o cruz" que muchas veces puede suponer un examen en fechas ordinarias. Esa ha sido la gran motivación para mi, el gran aliciente que me ha hecho trabajar cada día para reflexionar sobre lo hecho en clase en mis diarios, el saber que mi trabajo se vería recompensado en su justa medida.>> (HN44).

Evidentemente sería iluso por nuestra parte pensar que en tan solo un cuatrimestre y doce créditos de proyecto, podríamos contrarrestar la pedagogía venenosa $(22,23)$ inyectada en vena a nuestros alumnos a lo largo de 15 años de escolarización en un sistema jerarquizado y reproductor. Por eso no es de extrañar que muchos estudiantes no hayan sido capaces de captar la intencionalidad del diario de aprendizajes y lo hayan vivido como una actividad más y piensen que este no es $<<\ldots$ lo más significativo de todo este aprendizaje, ... Este ha sido el motivo por el cual lo he dejado un poco más de lado y me he centrado más en lo demás y no me ha dado tiempo a completar la parte de trabajo autónomo. > (HN75) En la otra cara de la moneda nos encontremos con estudiantes que si han sabido captar la filosofía de la herramienta y ver que es un aspecto fundamental para el proyecto, ya que $<<\ldots$.es un elemento mediante el cual podemos destacar aspectos de cada actividad tanto de clase como individual así como llevar un propio control de nuestra participación en la asignatura.> (HS75) Este comentario recoge perfectamente la doble intencionalidad que hemos querido dar a este diario, el de ser un instrumento para el docente de evaluación de las propuestas de enseñanza y aprendizaje que ha puesto en práctica y por otro lado que le sirva al estudiante para que, durante y al final del proceso del proyecto tome conciencia de su evolución y de las tareas que ha ido realizando para la mejora de sus competencias personales e instrumentales.

\section{CINCO APUNTES PARA LA REFLEXIÓN}

Sintetizando las ideas que hemos venido desgranado en la exposición, podemos concretar que:

No podemos pensar en la posibilidad de uniformar la metodología universitaria en base a las nuevas 
RIVERA, E. \& TRIGUEROS, C. El Diario de Aprendizajes. Un Instrumento de Ayuda en la Autoevaluación del Estudiante. Mot. Hum. 13(2); 110-117: 2012.

demandas del ECTS. La heterogeneidad que existente en las universidades siempre nos obligará a buscar las mejores soluciones en base a la diversidad del contexto en el que nos encontremos.

Lo que si se hace necesario es un cambio de mentalidad en el profesorado $y$ en los responsables universitarios. Los primeros deben tomar conciencia que la docencia requiere de tiempos de dedicación que hasta ahora, salvo excepciones, no se están produciendo. Hay que abandonar el individualismo para ser capaces de trabajar en colaboración y transformar la suficiencia en humildad para aprender a ser mejores docentes. En cuanto a los responsables universitarios es imprescindible que sitúen a la docencia en igualdad con la investigación. Además, es preciso invertir esfuerzos en bajar las ratios y mejorar infraestructuras.

La experiencia de nueve años con nuestro proyecto nos dice que el estudiante responde y se compromete cuando ve compromiso en el docente. Hay que abandonar el victimismo cómodo en el que estamos instalados y plantear propuestas de intervención en el aula que lleven a los estudiantes a vivir en la práctica lo que predicamos desde la teoría.

En cuanto a la herramienta del diario de aprendizaje, en líneas generales se ha manifestado como útil para el estudiante y el docente. A los primeros les ayuda a reflexionar y a mantener una actitud responsable ante el proyecto. A los docentes nos ha servido como sistema de autorregulación para ir adaptando la propuesta a las demandas y necesidades de nuestro alumnado. Por último queremos realizar una advertencia. Las herramientas son sólo herramientas y no podemos inferir que su utilización per se, mejora la docencia. Los cambios se producen desde las finalidades que nos planteamos desde la mediación en el proceso de enseñanza y aprendizaje, por lo que todos los elementos del proceso: principios, objetivos, contenidos, metodología y evaluación deben estar en íntima comunión si deseamos que al final el proyecto funcione.

\section{REFERNCIAS BIBLIOGRAFICAS}

1. Bologna Working Group On Qualifications Frameworks. A Framework for Qualifications of the European Higher Education Area. Copenhagen (DK): Ministry of Science,Technology and Innovation. CrédEur.doc]. (2005); Disponible en http://www.med.ub.es/ aprats/sae/docencia/creditosECTS. ppt. (Consultado el 10/09/2012)

2. Huber, G. L. Aprendizaje activo y metodologías educativas. En Revista de Educación, número extraordinario; .2008: 59-81

3. Shuell,T. J. Cognitive conceptions of learning. En Review of Educational Research, 1986; 56, 411-436.

4. Rodríguez, R. M. Innovación metodológica docente en el marco del espacio Europeo de Educación Superior: Algunas reflexiones desde los retos de la sociedad del conocimiento. En XXI, Revista de Educación, 2009; 11, 195-206

5. Masjuan, J. M. Convergencia europea, reformas universitarias, actitudes y prácticas de los estudiantes. En Educar; 2004; 33: 59-76

6. De La Torre, E., Rivera, E., Trigueros, C. Creencias y concepciones de la educación física en evolución: el caso de la formación del profesorado de educación física en la educación primaria. Revista Tándem. Didáctica de la Educación Física, 2007;23: 50-56

7. Rivera, E., De La Torre, E. Democratizar el aula universitaria: una propuesta alternativa de formación inicial universitaria desde la participación del alumnado. En Investigación en la Escuela, 2005; 57, 85-95.

8. Rivera, E., De La Torre, E., Trigueros, C. Formar docentes, formar personas: la formación inicial del profesorado desde una propuesta sociocrítica. Ciclo sobre Complejidad y Modelo Pedagógico. 2009. Disponible en http://www.tendencias21.net/ciclo/Un-centro-paraformacion-en-lacomplejidad- de-educadores-y-docentesdara-continuidad-al-Ciclo_a68.html] [Consultado el 02/07/2011]

9. Rivera, E.; Trigueros, C.; De La Torre, E., Moreno, A. Formar docentes, formar personas: una experiencia transdisciplinar para democratizar el aula universitaria. En L'Activitat del Docent: Intervenció, Innovació, Investigació. Barcelona: CiDd: II Congrés Internaiconal de Didáctiques, 2010; 1 - 8 .

10. De Lavigne, R. Créditos ECTS y métodos para su asignación. 2003. Disponible en http://www.uv.es/ oce/Asignacion_Espaol.pdf. (Consultado el: 01-07-2011). 
RIVERA, E. \& TRIGUEROS, C. El Diario de Aprendizajes. Un Instrumento de Ayuda en la Autoevaluación del Estudiante. Mot. Hum. 13(2); 110-117: 2012.

11. Barbosa, J., Guiteras, J., Fonrodona, G. Teaching chemistry in Europe - a look at future developments. En Anal Bioanal Chem, 2004; (378): 33-36.

12. Pagani, R. Convergencia de Programas al Crédito Europeo (ECTS). En Jornadas de docencia. 2002. Facultad de Medicina UCM.

13. Valero García, M. ¿Cómo nos ayuda el Tour de Francia en el diseño de programas docentes centrados en el aprendizaje? En Jornadas sobre Enseñanza Universitaria de la Informática. Universidad Politécnica de Cataluña. 2003. Disponible en http://www.eees.ua.es/conferencias/articulo _ECTS_Politecnica.pdf [Consultado el 03-07-2011]

14. Julián, J.; Zaragoza, J.; Castejón, F.J., López, V.M. Carga de trabajo en diferentes asignaturas que experimentan el sistema ECTS. En Revista Internacional de Medicina y Ciencias de la Actividad Física y el Deporte 2010; 10 (38): 218-233.

15. Barceló, O, Olivan, J., Asensio, A., González, J. A. Análisis del trabajo no presencial utilizando un enfoque ETCS. En Revista Complutense de Educación. 2007; 18 (1): 181-198.

16. Mikel Garmendia, M, Guisasola, J., Barragués, J. I., Zuza, K. ¿Cuánto tiempo dedican los estudiantes al estudio de asignaturas básicas de $1^{\circ}$ de ingeniería? En Didáctica de las Ciencias Experimentales y Sociales. 2006; 20: 89103.

17. Palomares, A., Garrote, D. Un nuevo modelo docente por y para el alumnado. En ENSAYOS, Revista de la Facultad de Educación de Albacete, 2009; 24: 2009. http://www.uclm.es/ab/educacion/ensayos - [Consultada el 02-07-2011]

18. López-Aguado, M. Diseño y análisis del Cuestionario de Estrategias de Trabajo Autónomo (CETA) para estudiantes universitarios. En Revista de Psicodidáctica, 2010; 15 (1): 77-99

19. Alonso, J. Motivación y aprendizaje en el aula. Cómo enseñar a pensar. Madrid: Santillana; (1995).

20. Apter, M. J. Motivational styles in everyday life: A guide to reversal theory. Washington: American Psychological Association; (2001).

21. Rinaudo, M. C., Donolo, D. Motivación y uso de estrategias en estudiantes universitarios. Su evaluación a partir del Motivated Strategies Learning Questionnaire En Anales de Psicología, 2003;19(1):107-119.

22. Fernández-Balboa, J. M. La autoevaluación como práctica promotora de la democracia y la dignidad. En Sicilia, A y Fernández Balboa, J. M. (coord.) La otra cara de la enseñanza: La Educación Física desde una perspectiva crítica. Barcelona: INDE. 2005; P: 127-158.
23. Miller, A. For your own good. New York: The Noonday Press. 1980.

\section{Dirigir Correspondencia a:}

Enrique Rivera García

erivera@ugr.es

C.P. 18071 - Granada España

(0034) 958249641

RECIBIDO: $30-11-2012$

ACEPTADO: $27-12-2012$ 